\title{
Ultrasound in Anesthetic Practice
}

\author{
Graham Arthurs, Barry Nicholls (Editors). Cambridge University Press, 2009. \\ Category: Textbook on Ultrasound, CD included. ISBN 978-0-521-71623-9
}

\author{
Marie-Andrée Girard, MD
}

Published online: 15 January 2010

(c) Canadian Anesthesiologists' Society 2009

Ultrasound in the specialty of anesthesiology has blossomed over the past half decade, with an ever-increasing role in the day-to-day care of patients in the perioperative setting. Lectures, case presentations, and workshops on ultrasound guidance for regional anesthesia and related indications have become ubiquitous at nearly every anesthesia continuing medical education meeting and scientific conference and within our teaching environment. Drs. Arthurs and Nicholls from the UK provide a new offering on this subject matter. Their textbook addresses the use of ultrasound in anesthetic practice and includes contributions from 24 contributing authors who are experts in their respective fields.

The 13 chapters of this book cover the field of ultrasound techniques across the entire practice of anesthesiology and critical care. Beginning with a well-written introduction that describes the relevant scientific elements related to ultrasound technology, the authors explain clearly the physics of ultrasound, the machines, and the ultrasound probes. In the ensuing chapters, a number of clinical scenarios ranging from emergency to non-emergency type cases are reviewed where ultrasound guidance is indicated. The authors have categorized chapters in a manner that reflects common clinical applications. Several chapters are organized according to the clinical setting (e.g., ultrasound in the intensive care unit and ultrasound for the unstable patient, the trauma patient, and those with soft tissue injury), according to modality (e.g., transesophageal echo, pregnancy echo, echocardiography, and ophthalmic echo), or according to anesthesia technique (e.g., regional nerve blocks for adults and children and regional nerve blocks for

M.-A. Girard, MD ( $\square)$

University of Sherbrooke, Sherbrooke, Canada

e-mail: marie-andree.girard@usherbrooke.ca vascular access procedures). This method of content organization allows the reader to cross-reference ultrasound techniques across various clinical applications. The authors begin each chapter with a brief description of the relevant underlying ultrasound technology, the related anatomy, and the unique equipment and technical requirements related to that chapter's subject. A sampling of specific chapter titles includes: "Use of ultrasound to aid local anesthetic nerve blocks in adults", "Ultrasound in the ICU" and "Ultrasound in the traumatized patient". Each chapter includes supporting illustrations and an up-to-date list of supporting references. The book concludes with a chapter on the assessment of soft tissue.

Overall, this book is well written; however, readers will observe that the organization within each chapter tends to be somewhat uneven, especially in the chapters entitled "Ultrasound in ophthalmic anesthesia" and "Use of ultrasound in the traumatized patient and the acute abdomen". This book also comes with an accompanying Compact Disc (CD). The CD component is compatible with either MAC or $\mathrm{PC}$ systems and is easy to use. The figures in the textbook are repeated in the $\mathrm{CD}$, allowing the reader to zoom in while also providing additional detailed illustrations specific to each block technique. While the image quality of some of the $\mathrm{CD}$ images is suboptimal, overall, the CD serves as a valuable aid for the reader to better understand the techniques that are presented.

Although Ultrasound in Anesthetic Practice is not as complete or as well illustrated compared with other recently published texts in this field, including Dr. Tsui's Atlas of ultrasound and nerve-stimulation-guided regional anesthesia (Springer, 2007) or Dr. Eisenberg's Échographie en anesthésie régionale périphérique (Arnette Blackwell, 2007), it is more comprehensive in other respects. A distinguishing element of Ultrasound in Anesthetic Practice 
is the fact that the book covers the field of echography in the trauma context as well as in regional anesthesia.

At first glance, it would appear that Ultrasound in Anesthetic Practice is written mostly by and for a broad base of anesthesiologists. The authors, who are mostly from the UK, present an extensive review of the use of ultrasound in a broad clinical context that is relevant to a large readership. While the multidisciplinary authorship includes important contributions from specialists in radiology, certain chapters will interest some anesthesiologists more than others. Anesthesiologists involved in critical care medicine will appreciate several chapters especially relevant to their discipline. In the perioperative setting, anesthesiologists will appreciate the range of ultrasound applications that the authors have covered. The book offers unique elements to facilitate learning for residents and students alike.

In conclusion, Ultrasound in Anesthetic Practice provides a reasonably comprehensive resource of the technology and techniques of ultrasound relevant to practicing anesthesiologists and critical care specialists, whereas more experienced echographers may be somewhat disappointed. Despite several noted limitations, this text remains a reasonable addition to any anesthesia departmental library. 